\title{
Controle por Modos Deslizantes de um Atuador Eletro-hidráulico com Compensação por Processo Gaussiano
}

\author{
Gabriel S. Lima* Wallace M. Bessa* \\ * RoboTeAM - Robotics $\&$ Machine Learning Universidade Federal do \\ Rio Grande do Norte, RN, (e-mail: limagabriel@ufrn.edu.br, \\ wmbessa@ct.ufrn.br).
}

\begin{abstract}
The development of precise control systems for electro-hydraulic actuators (EHA) depends on adequate compensation of the unknown dynamic effects. In this work, a Sliding Modes controller is combined with a Gaussian Process compensator to provide adequate trajectory tracking. Gaussian process is a well-known machine learning strategy that can be used in the recognition of functions. The closed-loop convergence properties of the system are analyzed by the Lyapunov Stability Theory. Numerical results confirm a strong improvement in the performance of the controller when the proposed compensator is inserted.

Resumo: O desenvolvimento de sistemas de controle precisos para atuadores eletro-hidráulicos depende de uma adequada compensação dos efeitos dinâmicos desconhecidos. Neste trabalho, um controlador por Modos Deslizantes é combinado com um compensador por Processo Gaussiano para proporcionar um adequado rastreamento de trajetória. Processo Gaussiano é uma conhecida estratégia de aprendizagem de máquinas que pode ser utilizada no reconhecimento de funções. As propriedades de convergência do sistema em malha fechada são analisadas pela Teoria de Estabilidade de Lyapunov. Resultados numéricos confirmam uma forte melhora na desempenho do controlador ao ser inserido o compensador proposto.
\end{abstract}

Keywords: control systems, electrohydraulic actuators; Sliding Modes; Gaussian Process; machine learning.

Palavras-chaves: sistemas de controle; atuadores eletro-hidráulicos; Modos Deslizantes; Processo Gaussiano; aprendizagem de máquina.

\section{INTRODUÇÃO}

Os atuadores eletro-hidráulicos (EHA, do inglês ElectroHydraulic Actuator) têm sido utilizados em inúmeras aplicações em virtude de não só poder aplicar forças e torques elevados, mas de também possuir uma alta razão potência/peso (Sun and Chiu, 1999; Liu and Alleyne, 1999; Yim et al., 2014). Esses dispositivos podem tanto estar incorporados a sistemas automotivos (Balau et al., 2011) quanto aplicados em exoesquelestos (Yang et al., 2018) ou no campo aeroespacial (Altare and Vacca, 2015).

Por conta das vantagens mencionadas dos EHA, em aplicações que exigem alta precisão de operação faz-se necessário desenvolver um controlador capaz de fornecer um bom desempenho. Entretanto, os EHA possuem um alto grau de não-linearidade por conta dos efeitos da compressibilidade do fluido, atuação da válvula, atrito, saturação, zona morta, entre outros (Bessa et al., 2010; Yim et al., 2014). Em relação aos controladores já implementados para sistemas eletro-hidráulicos a literatura apresenta diversos exemplos com técnicas de controle e compensadores para lidar com as não-linearidades presentes nos atuado-

\footnotetext{
* Os autores agradecem a Coordenação de Aperfeiçoamento de Pessoal de Ensino Superior (CAPES) e ao Conselho Nacional de Desenvolvimento Científico e Tecnológico (CNPq).
}

res. Bonchis et al. (2002) compararam experimentalmente diversas técnicas de controle, entre elas o ProporcionalDerivativo (PD) e controle preditivo. Bessa et al. (2010) utilizaram Lógica Fuzzy como estratégia adaptativa, dentro de um controlador por SMC (do inglês Sliding Mode Control), para estimar as incertezas devido ao efeito de uma zona morta desconhecida no esforço de controle. Skarpetis and Koumboulis (2013) aplicaram uma estratégia robusta a um controlador Proporcional-Integral-Derivativo (PID). E Liem et al. (2016) também usaram o controlador PID como controlador aliado dessa vez com Redes Neurais em um contexto feedforward para melhorar o desempenho do controlador.

Nesse trabalho, um novo tipo de compensador (ou estimador de incertezas) é apresentado dentro de um controlador robusto. Ele se baseia no Processo Gaussiano (GP, do inglês Gaussian Process). O compensador GP vem dentro do contexto do problema de regressão (GPR, do inglês Guassian Process Regression) de funções, no caso da dinâmica desconhecida do sistema, das perturbações.

O GP tem sido aplicado nas mais diversas situações. Por exemplo, dentro de machine learning, Doerr et al. (2017) tem utilizado GP para aprender a dinâmica do sistema aplicando-o ao problema de aperfeiçoamento de robustez de um modelo baseado em aprendizagem por reforço. 
Xiloyannis et al. (2017) também têm usado GP para substituir a relação entre atividade muscular e movimento pretendido de uma mão por uma abordagem que leva em conta movimentos prévios e ação muscular instântanea da mão para prever movimentos futuros. Já GPR tem sido aplicado para modelar a dinâmica de corpo rígido utilizando como dados de entrada os ângulos de Euler (Lang and Hirche, 2017). Doerr et al. (2018) mostra como a GPR pode ser adotada como um modelo não-paramétrico para estimar as incertezas da planta. Por fim, GPR com lei de controle robusta por Modos Deslizantes já vem sendo utilizado para o projeto de controlador de um motor a diesel (Aran and Unel, 2018). Entretanto, diferentemente da metodologia deste último trabalho, aqui o treinamento do GP será feito online.

O controlador robusto a ser utilizado nesse trabalho será o controlador por Modos Deslizantes. A análise de estabilidade desse controlador será feita pela Teoria de Lyapunov. Simulações numéricas com a implementação desse controlador SMC com a compensação por GPR serão feitas para um modelo de um atuador eletro-hidráulico a fim de verificar o desempenho desse sistema de controle.

\section{MODELO DO SISTEMA}

Considere a representação esquemática do sistema eletrohidráulico mostrada na Fig. 1.

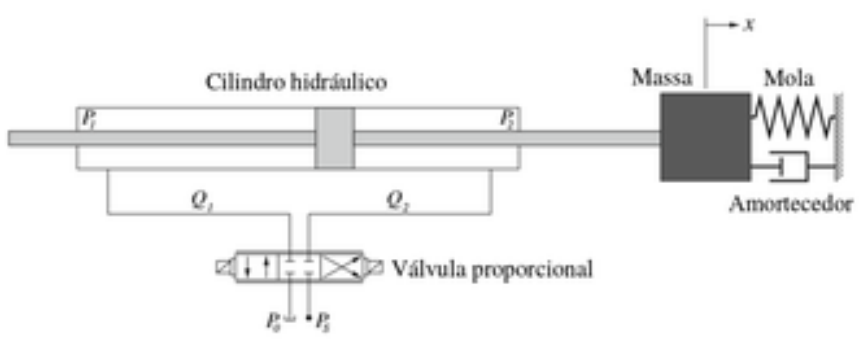

Figura 1. Representação esquemática do atuador eletrohidráulico, adaptado de Bessa et al. (2010)

O sistema considerado é formado por um cilindro hidráulico, uma válvula proporcional de quatro vias e um sistema massa-mola-amortecedor para representar a carga variável submetida. Aplicando a $2^{\text {a }}$ Lei de Newton no pistão chegase,

$$
F_{r}=A_{1} P_{1}-A_{2} P_{2}=M \ddot{x}+B \dot{x}+K x
$$

onde, $F_{r}$ é a força resultante atuando no pistão, $P_{1}$ e $P_{2}$ são as pressões atuantes em cada lado da câmara do cilindro, $A_{1}$ e $A_{2}$ são as áreas de cada câmara, $M$ é a massa total do pistão e da carga submetida, $B$ é o coeficiente de amortecimento viscoso do pistão e da carga, $K$ é a rigidez da mola e $x$ é o deslocamento do pistão.

Admitindo que a haste seja passante no pistão, então $A_{p}=A_{1}=A_{2}$. Logo, a diferença de pressão no cilindro poderá ser escita na forma $P_{q}=P_{1}-P_{2}$. Com isso, (1) pode ser reescrita na seguinte forma:

$$
A_{p} P_{q}=M \ddot{x}+B \dot{x}+K x
$$

Pela Equação da Continuidade, o fluxo do fluido hidráulico pode ser descrito por,

$$
Q_{l}=A_{p} \dot{x}+C_{t p} P_{q}+\frac{V_{t}}{4 \beta_{e}} \dot{P}_{q}
$$

onde, $Q_{l}$ é a vazão média que entra em ambas as câmaras, $C_{t p}$ é o coeficiente de vazamento do fluido que ocorre devido a folga entre o pistão e o cilindro, $V$ é o volume total das câmaras e $\beta_{e}$ é o fator de compressibilidade do fluido.

Considerando que pressão de retorno é muito menor que as demais pressões envolvidas $\left(P_{0} \approx 0\right)$, a vazão do fluído pode ser descrita pela seguinte equação,

$$
Q_{l}=C_{d} \omega \bar{x}_{s p} \sqrt{\frac{P_{s}-\operatorname{sgn}\left(\bar{x}_{s p}\right) P_{q}}{\rho}}
$$

onde $C_{d}$ é o coeficiente de descarga, $\omega$ é o gradiente de área do orifício da válvula, $\bar{x}_{s p}$ é o deslocamento efetivo da válvula, $P_{s}$ é a pressão de alimentação e $\rho$ é a massa específica do fluido.

Pode-se considerar que a tensão de controle $(u)$ é proporcional a $\bar{x}_{s p}$ assumindo que a dinâmica da válvula é tão rápida a ponto de ser desprezada.

Bessa et al. (2010) adicionam como não-linearidade ao EHA o efeito de zona morta. Santos (2018) justifica essa zona morta pelo fato dela permitir que a posição do cilindro seja mantida constante devido ao não vazamento do fluido durante a mudança de posição da válvula. Dito isso, o deslocamento efetivo da válvula pode ser representado pela seguinte expressão, considerando o efeito de zona morta,

onde,

$$
\bar{x}_{s p}=k_{v}(u)[u-d(u)]
$$

$$
k_{v}(u)=\left\{\begin{array}{c}
k_{l}, u \leq 0 \\
k_{r}, u>0
\end{array}\right.
$$

e,

$$
d(u)=\left\{\begin{array}{l}
\delta_{l}, \quad u \leq \delta_{l} \\
u, \delta_{l}<u<\delta_{r} \\
\delta_{r} \quad u \geq \delta_{r}
\end{array}\right.
$$

Combinando (2-5) chega-se em uma equação diferencial de terceira ordem que representa a dinâmica do EHA:

$$
\dddot{x}=-\boldsymbol{a}^{\top} \boldsymbol{x}+b(\boldsymbol{x}, u)[u-d(u)]
$$

onde $\boldsymbol{x}=[x, \dot{x}, \ddot{x}]^{\top}$ é o vetor de estados cujos coeficientes $\boldsymbol{a}=\left[a_{0}, a_{1}, a_{2}\right]^{\top}$ são dados por:

$$
\begin{gathered}
a_{0}=\frac{4 \beta_{e} C_{t p} K}{V_{t} M} \\
a_{1}=\frac{K}{M}+\frac{4 \beta_{e} A_{p}^{2}}{V_{t} M}+\frac{4 \beta_{e} C_{t p} B}{V_{t} M} \\
a_{2}=\frac{B}{M}+\frac{4 \beta_{e} C_{t p}}{V_{t}}
\end{gathered}
$$

e,

$$
\begin{aligned}
b(\boldsymbol{x}, u) & =\frac{4 \beta_{e} A_{p}}{V_{t} M} C_{d} \omega k_{v}(u) \times \\
& \times \sqrt{\frac{1}{\rho}\left[P_{s}-\frac{\operatorname{sgn}(u)(M \ddot{x}+B \dot{x}+K x)}{A_{p}}\right]}
\end{aligned}
$$

\section{PROCESSO GAUSSIANO}

Um GP pode ser definido como uma distribuição de probabilidades sobre o espaço de funções $h$ desde que um conjunto finito de entrada e saída, isto é, $(\boldsymbol{x}, h(\boldsymbol{x}))$, possuam uma distribuição gaussiana conjunta (Williams and Rasmussen, 2006). Por se tratar de uma distribuição 
de probabilidades, um GP é especificado através de suas funções média $\mu$ e de covariância $k$ a piori:

$$
h(\boldsymbol{x}) \sim \mathcal{G P}\left(\mu(\boldsymbol{x}), k\left(\boldsymbol{x}, \boldsymbol{x}^{\prime}\right)\right)
$$

onde, a média e variância são definidas como,

$$
\begin{aligned}
\mu(\boldsymbol{x})=\mathbb{E}[h(\boldsymbol{x})] \\
k\left(\boldsymbol{x}, \boldsymbol{x}^{\prime}\right)=\mathbb{V}\left(\boldsymbol{x}, \boldsymbol{x}^{\prime}\right) \\
=\mathbb{E}\left[(h(\boldsymbol{x})-\mu(\boldsymbol{x}))\left(h\left(\boldsymbol{x}^{\prime}\right)-\mu\left(\boldsymbol{x}^{\prime}\right)\right)\right]
\end{aligned}
$$

GPR consiste em estabelecer um modelo não-paramétrico para estimar funções desde que possam ser descritas através de um GP. Para tanto, considere que a função $h$ a ser estimada por GPR possa ser representada na seguinte forma:

$$
\bar{h}(\boldsymbol{x})=h(\boldsymbol{x})+\varepsilon, \quad \varepsilon \sim \mathcal{N}\left(0, \sigma_{\epsilon}^{2}\right)
$$

cujo o ruído $\varepsilon$ segue uma distribuição normal com média nula e variância $\sigma_{\epsilon}^{2}$.

O processo de aprendizagem por GPR consiste em, dado um conjunto de treinamento $\mathcal{D}_{N}=\left\{\boldsymbol{x}_{i}, h_{i}\right\}_{i=1}^{N}$, estimar o valor da função $h^{*}=h\left(\boldsymbol{x}^{*}\right)$ para entradas arbitrárias $\boldsymbol{x}^{*}$. Lima et al. (2018) e Doerr et al. (2018) sugerem que tanto a média quanto a variância a posteriori sejam calculadas com base nas seguintes equações:

$$
\begin{gathered}
\mathbb{E}\left(h^{*} \mid \mathcal{D}_{N}\right)=\mu\left(\boldsymbol{x}^{*}\right)+ \\
k\left(\boldsymbol{x}^{*}, \boldsymbol{x}\right)\left(\boldsymbol{K}_{N}+\sigma_{\epsilon}^{2} \boldsymbol{I}\right)^{-1} \tilde{h}(\boldsymbol{x}) \\
\mathbb{V}\left(h^{*} \mid \mathcal{D}_{N}\right)=k\left(\boldsymbol{x}^{*}, \boldsymbol{x}^{*}\right)- \\
k\left(\boldsymbol{x}^{*}, \boldsymbol{x}\right)\left(\boldsymbol{K}_{N}+\sigma_{\epsilon}^{2} \boldsymbol{I}\right)^{-1} k\left(\boldsymbol{x}, \boldsymbol{x}^{*}\right)
\end{gathered}
$$

onde $\boldsymbol{K}_{N}$ é definida por

$$
\boldsymbol{K}_{N}=\left[\begin{array}{ccc}
k\left(\boldsymbol{x}_{1}, \boldsymbol{x}_{1}^{\prime}\right) & \ldots & k\left(\boldsymbol{x}_{1}, \boldsymbol{x}_{N}^{\prime}\right) \\
\vdots & \ddots & \vdots \\
k\left(\boldsymbol{x}_{N}, \boldsymbol{x}_{1}^{\prime}\right) & \ldots & k\left(\boldsymbol{x}_{N}, \boldsymbol{x}_{N}^{\prime}\right)
\end{array}\right]
$$

e $\tilde{h}(\boldsymbol{x})=\left[\bar{h}\left(\boldsymbol{x}_{1}\right)-\mu\left(\boldsymbol{x}_{1}\right), \bar{h}\left(\boldsymbol{x}_{2}\right)-\mu\left(\boldsymbol{x}_{2}\right), \ldots, \bar{h}\left(\boldsymbol{x}_{N}\right)-\right.$ $\left.\mu\left(\boldsymbol{x}_{N}\right)\right]^{\top} \cdot \bar{h}\left(\boldsymbol{x}_{i-1}\right)$ é a saída em relação a entrada $\boldsymbol{x}_{i-1}$ que pertence ao conjunto de treinamento e é utilizada para calcular a média a posteriori no instante $i$.

\section{CONTROLE POR MODOS DESLIZANTES}

Como o modelo do EHA é dado por um sistema de $3^{\text {a }}$ ordem, considere então que esse modelo pode ser descrito genericamente na seguinte forma:

$$
\dddot{x}(t)=f(\boldsymbol{x}, t)+b(\boldsymbol{x}, t) u(t)
$$

onde $\dddot{x}$ representa a terceira derivada em relação ao tempo da variável de estado $x$ e $u$ a variável de entrada, as funções $f$ e $b: \mathbb{R}^{3} \rightarrow \mathbb{R}$ são funções não-lineares.

Pelo método de controle por SMC (Slotine and Li, 1991), uma superfície dita de deslizamento no espaço de estados é capaz de realizar o mapeamento $s: \mathbb{R}^{3} \rightarrow \mathbb{R}$,

$$
s(\boldsymbol{x}, t)=\ddot{e}+2 \lambda \dot{e}+\lambda^{2} e
$$

onde $\lambda$ é uma constante estritamente positiva e $e=x-x_{d}$ é o erro de rastreamento.

Consideração 1. O termo $b$ é desconhecido, mas sempre positivo e limitado na seguinte forma:

$$
0<b_{\min }<b<b_{\max }
$$

Considera-se que o modelo do sistema possa ser separado em uma dinâmica conhecida $\hat{f}$ e outra desconhecida ou não-modelada tratada aqui como perturbação $d$. Logo, adaptando de (16):

$$
\dddot{x}(t)=\hat{f}(\boldsymbol{x}, t)+b(\boldsymbol{x}, t) u(t)+d(\boldsymbol{x}, t)
$$

Consideração 2. A perturbação $d$ é uma função desconhecida, mas limitada, ou seja, $|d| \leq \delta$.

A lei de controle do sistema então pode ser escrita da seguinte forma, com a utilização da função saturação para evitar o efeito de chattering sobre o sistema:

$$
u=\hat{b}^{-1}\left(-\hat{f}-\hat{d}+\dddot{x}_{d}-2 \lambda \ddot{e}-\lambda^{2} \dot{e}-\kappa \operatorname{sat}(s / \phi)\right)
$$

onde $\hat{b}$ é a estimativa do termo $b, \hat{d}$ é a estimativa da perturbação a ser estimada pelo GPR, $\kappa$ é um termo que garanta que o sistema alcance a superfície de deslizamento e $\phi$ é a espessura da camada limite.

Observação 1. Apesar da Consideração 1 não levar em conta formalmente o GP, que por ser uma distribuição de probabilidades possui, em tese, extensão infinita, em casos reais perturbações ilimitadas geralmente não ocorrem. Por conta disso, é possível estabelecer um intervalo cuja robustez do controlador seja garantida.

Consideração 3. O limitante $\delta$ da perturbação corresponde a um intervalo de $95 \%$ de confiança da distribuição obtida pelo GP, ou seja, $\delta=|\hat{d}|+2 \sigma_{d}$, onde $\sigma_{d}^{2}=\mathbb{V}\left(h^{*} \mid \mathcal{D}_{N}\right)$.

Pela Teoria da Estabilidade de Lyapunov é possível demonstrar as propriedades de convergência do sistema em malha fechada. Para isto, considere a seguinte função definida positiva:

$$
V(t)=\frac{1}{2} s_{\phi}^{2}
$$

onde $s_{\phi}$ é a medida da distância do estado atual até a camada limite $s_{\phi}=s-\phi \operatorname{sat}(s / \phi)$.

Sabendo que $\dot{s}_{\phi}=s_{\phi}=0$ dentro da camada limite e $\dot{s}_{\phi}=s_{\phi}$ fora dela, a derivada $\dot{V}$ pode ser escrita como

$$
\begin{aligned}
\dot{V}(t) & =s_{\phi} \dot{s}_{\phi}=\left(\dddot{x}-\dddot{x}_{d}+2 \lambda \ddot{e}+\lambda^{2} \dot{e}\right) s_{\phi} \\
& =\left(\hat{f}+b u+d-\dddot{x}_{d}+2 \lambda \ddot{e}+\lambda^{2} \dot{e}\right) s_{\phi}
\end{aligned}
$$

Considerando que a estimativa do termo $b$ pode ser calculada pela média geométrica dos seus respectivos limites, ou seja, $\hat{b}=\sqrt{b_{\max } b_{\min }}, \operatorname{logo}(18)$ pode ser reescrita da seguinte maneira:

$$
0<\nu^{-1}<b \hat{b}^{-1}<\nu
$$

onde $\nu=\sqrt{b_{\max } / b_{\min }}$.

Aplicando a lei de controle (20) em (22) e notando que fora da camada limite $\operatorname{sat}(s / \phi)=\operatorname{sgn}\left(s_{\phi}\right)$, pode-se definir o ganho do controlador como

$$
\kappa \leq \nu(\eta+\delta)+|\hat{d}|+(\nu-1)\left|-\hat{f}+\dddot{x}_{d}-2 \lambda \dot{e}-\lambda^{2} e\right|
$$

para garantir que,

$$
\dot{V}(t) \leq-\eta\left|s_{\phi}\right|
$$

que implica $V(t) \leq V(0)$ e que $s_{\phi}$ é limitado. Uma vez dentro da camada limite conclui-se que $|s| \leq \phi$. Logo, pode-se demonstrar que o erro de rastreamento converge para uma região fechada definida por $\Phi=$ $\left\{\boldsymbol{e} \in \mathbb{R}^{3}|| e^{(i)} \mid \leq(i+1) ! \lambda^{i-2} \phi, i=0,1,2\right\}$, (Bessa, 2009). 


\section{DERIVADOR POR MODOS DESLIZANTES}

Para efeitos de simulação, foi considerado que apenas a posição do atuador era medida. Assim sendo, para obter os demais estados foi utilizado um derivador por Modos Deslizantes de $2^{\text {a }}$ Ordem (2-SMD, do inglês Second-order Sliding Mode Differentiator). Santos (2018) tem utilizado esse método para obter os estados necessários a partir de uma medida de posição em um caso aplicado a um EHA. Tanto do ponto de vista teórico (Levant, 2003) quanto experimental (Pisano and Usai, 2004) essa técnica de diferenciação dos estados apresentou ótimo desempenho.

Essa técnica consiste em estimar o sinal $\dot{\xi}_{0}$ de um sinal $x=f(t)$ de modo que a igualdade $\xi_{0}-x=0$ seja válida $\forall t \geq t_{\xi_{0}}$ dentro de um intervalo de convergência $0 \leq t \leq t_{\xi_{0}}$. Assim sendo, definindo o erro $\tilde{\xi}=\xi_{0}-x$, a derivada de $f(t)$ pode ser estimada através das seguintes equações:

$$
\begin{gathered}
\dot{\xi}_{0}=\xi_{1}-\varrho_{0}|\tilde{\xi}|^{\frac{1}{2}} \operatorname{sgn}(\tilde{\xi}) \\
\dot{\xi}_{1}=-\varrho_{1} \operatorname{sgn}(\tilde{\xi})
\end{gathered}
$$

onde a convergência do algoritmo será dada quando,

$$
\begin{gathered}
\varrho_{1}>\mathcal{C} \\
\varrho_{0}^{2}>4 \mathcal{C} \frac{\varrho_{1}+\mathcal{C}}{\varrho_{1}-\mathcal{C}}
\end{gathered}
$$

onde $\mathcal{C}$ é uma constante associada a função de Lipschitz $\dot{f}$.

Definição. Uma função $f: \mathcal{X} \rightarrow \mathbb{R}$ é dita de Lipschitz se existir uma constante $\mathcal{C}>0$, tal que $x, y \in \mathcal{X} \rightarrow \mid f(x)-$ $f(y)|\leq \mathcal{C}| x-y \mid($ Lima, 2004).

\section{SIMULAÇÃO}

As simulações foram computadas em linguagem $\mathrm{C}++$ com taxas de amostragem do controlador e do simulador de 100 $\mathrm{Hz}$ e $500 \mathrm{~Hz}$, respectivamente. O Método de Runge-Kutta de $4^{\text {a }}$ Ordem foi utilizado para resolver numericamente o modelo do sistema.

\begin{tabular}{|c|c|}
\hline Parâmetro & Valor \\
\hline$P_{s}$ & $7 \mathrm{MPa}$ \\
\hline$\rho$ & $850 \mathrm{~kg} / \mathrm{m}^{3}$ \\
\hline$C_{d}$ & 0.6 \\
\hline$\omega$ & $2.5 \cdot 10^{-2} \mathrm{~m}$ \\
\hline$A_{p}$ & $3 \cdot 10^{-3} \mathrm{~m}^{2}$ \\
\hline$C_{t p}$ & $2 \cdot 10^{-12} \mathrm{~m}^{3} /(\mathrm{s} . \mathrm{Pa})$ \\
\hline$\beta_{e}$ & $700 \mathrm{MPa}$ \\
\hline$V_{t}$ & $6 \cdot 10^{-4} \mathrm{~m}^{3}$ \\
\hline$M_{t}$ & $20 \mathrm{~kg}$ \\
\hline$B$ & $100 \mathrm{Ns} / \mathrm{m}$ \\
\hline$K$ & $75 \mathrm{~N} / \mathrm{m}$ \\
\hline$k_{l}$ & $1.8 \cdot 10^{-6} \mathrm{~m} / \mathrm{V}$ \\
\hline$k_{r}$ & $2.2 \cdot 10^{-6} \mathrm{~m} / \mathrm{V}$ \\
\hline$\delta_{l}$ & $-0.5 \mathrm{~V}$ \\
\hline$\delta_{r}$ & $0.5 \mathrm{~V}$ \\
\hline
\end{tabular}

Os parâmetros adotados para o sistema eletro-hidráulico estão mostrados na Tabela 1.

Tabela 1. Parâmetros de simulação.

Para o controlador foram considerados os parâmetros $\lambda=8, \phi=0.1, \nu=3$ e $\eta=0.5$. A estimativa do termo $b$ foi posto $\hat{b}=1.0$ e também foi considerado que não possuíamos conhecimento prévio do termo $f$, ou seja, $\hat{f}=0$.

Para o GPR, foi considerado como entrada os estados $\boldsymbol{x}=\left[\begin{array}{lll}x & \dot{x} & \ddot{x}\end{array}\right]^{\top}$ e a média a priori nula $(\mu(s)=0)$. Como adotado por Lima et al. (2018), foi utilizado como função de covariância o kernel da Função de Fase Radial (RBF, do inglês Radial Basis Function) também chamada de exponencial quadrática, definida abaixo:

$$
k\left(s, s^{\prime}\right)=\sigma_{f}^{2} \exp \left(-\frac{\left\|s-s^{\prime}\right\|^{2}}{2 \gamma^{2}}\right)
$$

onde $\sigma_{f}^{2}$ e $\gamma$ são hiper-parâmetros. Os parâmetros considerados foram $\sigma_{\epsilon}=0.7, \sigma_{f}=3.0$ e $\gamma=100$. Foi posto que o atuador deveria seguir a trajetória $x_{d}=0.1[1-\cos (\pi t / 10)]$ por um tempo de $t_{\text {sim }}=60 \mathrm{~s}$.

Como é possível verificar pela Fig. 2, o controle proposto (SMC+GPR) para o atuador eletro-hidráulico foi capaz de melhorar o rastreamento de trajetória com pequeno erro de rastreamento se comparado com o controlador convencional (SMC), Fig. 4. Para tanto, a inclusão do compensador GP elevou o esforço de controle, Fig. 3. Pela Fig. 5 é possível visualizar a estimativa GPR aproximando a dinâmica desconhecida do sistema.

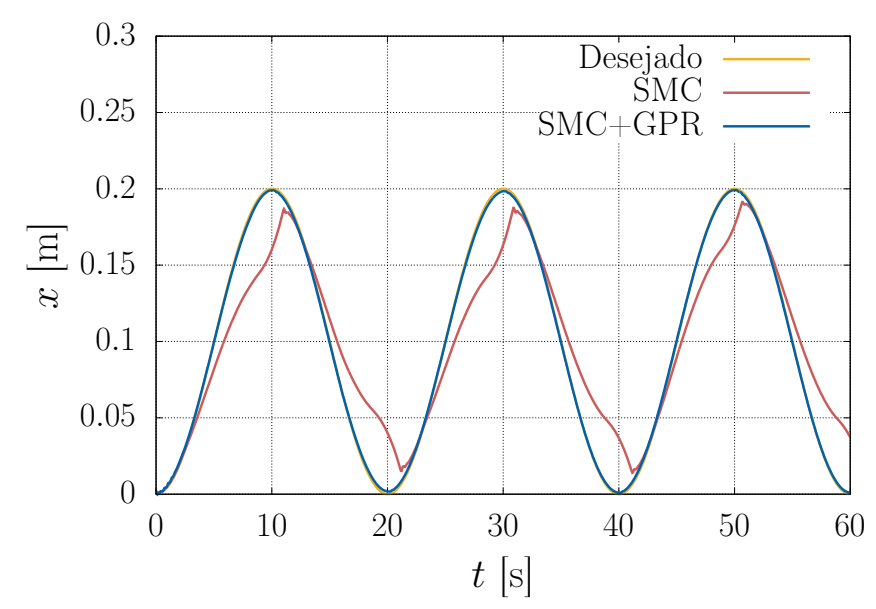

Figura 2. Rastreamento de trajetória.

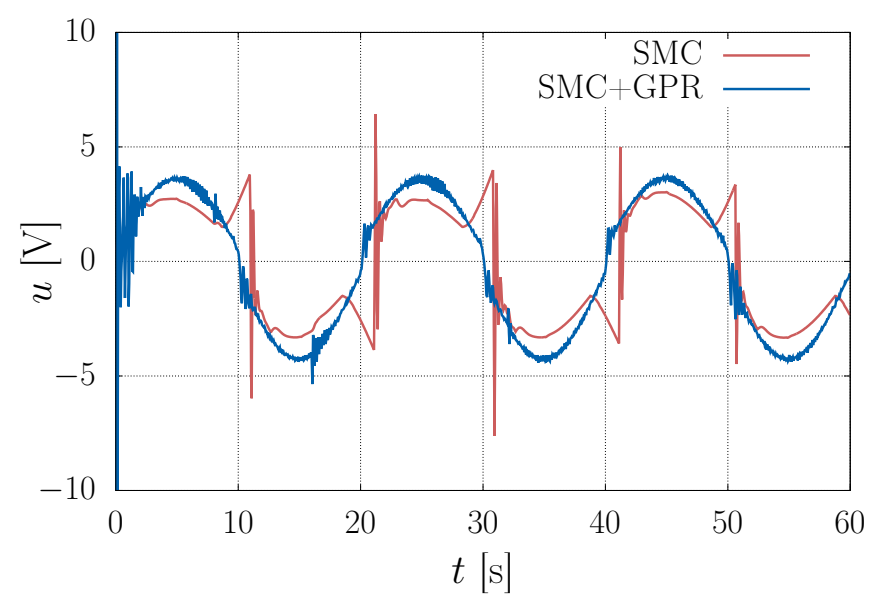

Figura 3. Esforço de controle. 


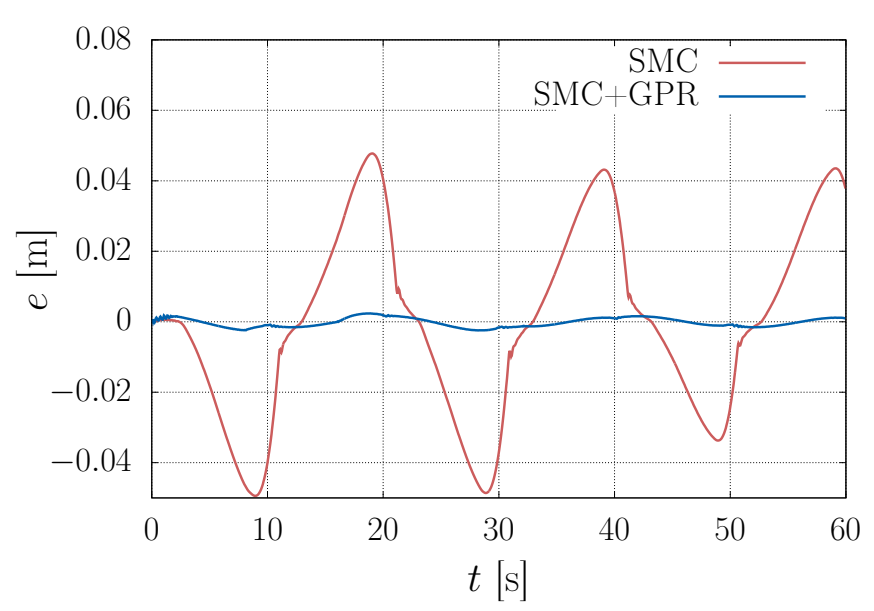

Figura 4. Erro de rastreamento.

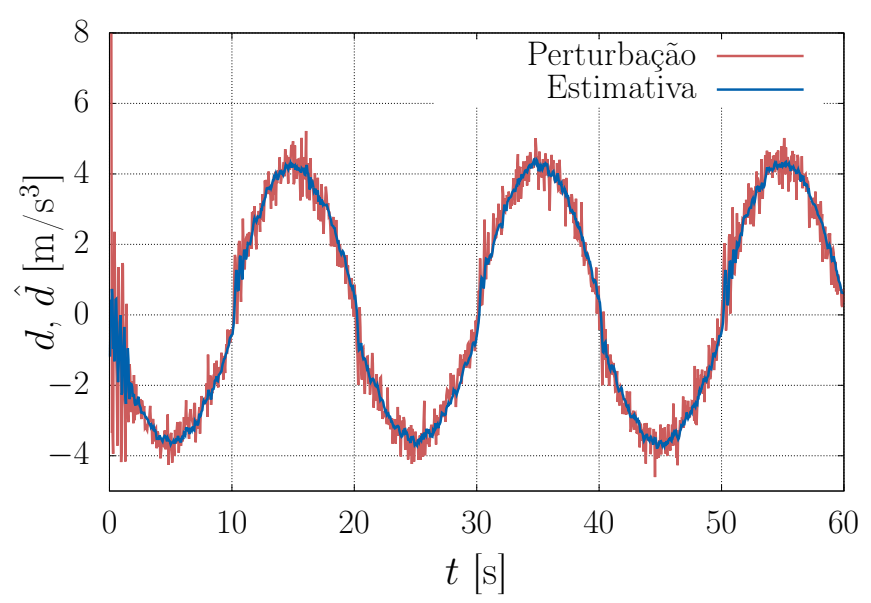

Figura 5. Perturbação e GPR.

\section{CONCLUSÕES}

Neste trabalho, um controlador por modos deslizantes é combinado com uma função de regressão por Processo Gaussiano com objetivo de realizar o rastreamento de trajetória de um atuador eletro-hidráulico. GPR é usado como um modelo não-paramétrico para compensar a dinâmica não modelada do sistema. O Teorema da Estabilidade de Lyapunov é utlizado para demonstrar as propriedades de convergência do sistema em malha-fechada. Através de simulações numéricas, é demonstrado uma significativa melhora no desempenho do controlador em comparação a abordagem convencional (SMC).

\section{AGRADECIMENTOS}

Os autores desse trabalho agradecem ao Programa de Pós-Graduação em Engenharia Mecânica da Universidade Federal do Rio Grande do Norte (PPGEM/UFRN), a Coordenação de Aperfeiçoamento de Pessoal do Ensino Superior (CAPES) e ao Conselho Nacional de Desenvolvimento Científico e Tecnológico ( $\mathrm{CNPq})$.

\section{REFERÊNCIAS}

Altare, G. and Vacca, A. (2015). A design solution for efficient and compact electro-hydraulic actuators.
Procedia Engineering, 106, 8-16.

Aran, V. and Unel, M. (2018). Gaussian process regression feedforward controller for diesel engine airpath. International Journal of Automotive Technology, 19(1), 635642. doi:10.1007/s12239.

Balau, A.E., Caruntu, C.F., and Lazar, C. (2011). Simulation and control of an electro-hydraulic actuated clutch. Mechanical Systems and Signal Processing, 25(6), 19111922.

Bessa, W.M., Dutra, M.S., and Kreuzer, E. (2010). Sliding mode control with adaptive fuzzy dead-zone compensation of an electro-hydraulic servo-system. Journal of Intelligent and Robotic Systems, 58(1), 3-16.

Bessa, W.M. (2009). Some remarks on the boundedness and convergence properties of smooth sliding mode controllers. International Journal of Automation and Computing, 6(2), 154-158. doi:10.1007/s11633-009-0154-z.

Bonchis, A., Corke, P.I., and Rye, D.C. (2002). Experimental evaluation of position control methods for hydraulic systems. IEEE Transactions on Control Systems Technology, 10(6), 876-882.

Doerr, A., Daniel, C., Nguyen-Tuong, D., Marco, A., Schaal, S., Marc, T., and Trimpe, S. (2017). Optimizing long-term predictions for model-based policy search. In Conference on Robot Learning, 227-238.

Doerr, A., Daniel, C., Schiegg, M., Nguyen-Tuong, D., Schaal, S., Toussaint, M., and Trimpe, S. (2018). Probabilistic Recurrent State-Space Models. In Proceedings of the 35th International Conference on Machine Learning, 1280-1289. Stockholm. doi:http://dx.doi.org/10.1111/j. 1479-828X.2009.00955.x.

Lang, M. and Hirche, S. (2017). Computationally efficient rigid-body gaussian process for motion dynamics. IEEE Robotics and Automation Letters, 2(3), 1601-1608.

Levant, A. (2003). Higher-order sliding modes, differentiation and output-feedback control. International journal of Control, 76(9-10), 924-941.

Liem, D.T., Truong, D.Q., Park, H.G., and Ahn, K.K. (2016). A feedforward neural network fuzzy grey predictor-based controller for force control of an electrohydraulic actuator. International Journal of Precision Engineering and Manufacturing, 17(3), 309-321.

Lima, E.L. (2004). Curso de análise, Volume 1. Projeto Euclides, IMPA, 11th edition.

Lima, G.S., Bessa, W.M., and Trimpe, S. (2018). Depth control of underwater robots using sliding modes and gaussian process regression. In IEEE LARS 2018 15th Latin American Robotics Symposium. IEEE, João Pessoa.

Liu, R. and Alleyne, A. (1999). Nonlinear force/pressure tracking of an electro-hydraulic actuator. IFAC Proceedings Volumes, 32(2), 952-957.

Pisano, A. and Usai, E. (2004). Output-feedback control of an underwater vehicle prototype by higher-order sliding modes. Automatica, 40(9), 1525-1531.

Santos, J.D.B. (2018). Compensação de atrito no controle de sistemas mecânicos : Uma abordagem utilizando estratégias inteligentes. Ph.D. thesis, Universidade Federal do Rio Grande do Norte, Natal.

Skarpetis, M.G. and Koumboulis, F.N. (2013). Robust pid controller for electro-hydraulic actuators. In Emerging Technologies 8 Factory Automation (ETFA), 2013 IEEE 18th Conference on, 1-5. IEEE. 
Slotine, J.J.E. and Li, W. (1991). Applied Nonlinear Control. Prentice-Hall, Inc., Englewood Cliffs, 1 edition.

Sun, H. and Chiu, G.C. (1999). Nonlinear observer based force control of electro-hydraulic actuators. In American Control Conference, 1999. Proceedings of the 1999, volume 2, 764-768. IEEE.

Williams, C.K. and Rasmussen, C.E. (2006). Gaussian processes for machine learning. the MIT Press, 2(3), 4.

Xiloyannis, M., Gavriel, C., Thomik, A.A., and Faisal, A.A. (2017). Gaussian Process Autoregression for Simultaneous Proportional Multi-Modal Prosthetic Control with Natural Hand Kinematics. IEEE Transactions on Neural Systems and Rehabilitation Engineering, 25(10), 1785-1801. doi:10.1109/TNSRE.2017.2699598.

Yang, Y., Huang, D., and Dong, X. (2018). Robust repetitive learning control of lower limb exoskeleton with hybrid electro-hydraulic system. In 2018 IEEE 7th Data Driven Control and Learning Systems Conference (DDCLS), 718-723. IEEE.

Yim, J., Kim, S., and Choi, Y. (2014). Adaptive torque control of hydraulic actuators based on dynamic compensation. In Ubiquitous Robots and Ambient Intelligence (URAI), 2014 11th International Conference on, 448-451. IEEE. 